\title{
A FORMAL METHOD FOR ANALYZING AND INTEGRATING THE RULE-SETS OF MULTIPLE EXPERTS
}

\author{
OJelanki K. NGWENYAMA ${ }^{1} \dagger$ and NOEL BrySON ${ }^{2}$ \\ 'Computer and Information Systems. School of Business Administration. The University of Michigan. \\ Ann Arbor. MI48109-1234, U.S.A. \\ 'Department of Information Systems and Analysis, School of Business Administration, \\ Howard University, washington D.C., U.S.A.
}

(Received 23 April 1990; in revised form 8 May 1991)

\begin{abstract}
Although there has been a movement toward the use of multiple sources of knowledge for expert systems development, there are no formal methods to guide knowledge engineers in integrating these sources. Further approaches for dealing with problems of inaccuracies, inconsistencies, and imcompleteness are not widely discussed in literature. This paper discusses a formal method for documenting, integrating and normalizing knowledge-bases derived from different knowledge sources. A case study is used to demonstrate the effectiveness of the method.
\end{abstract}

Kev words: Normalizing rule-bases, integrating rules, expert systems development

\section{INTRODUCTION}

Knowledge engineering is the sub-area of artificial intelligence which is concerned with expert systems development. During the development process, the contents of the knowledge component [the knowledge-base (KB)] must be defined. One of the primary objectives of knowledge engineering is to develop a complete, consistent and unambiguous description of the $\mathrm{KB}$. The traditional approach to KB definition is a one-on-one interaction and dialogue between the knowledge engineer and a single domain expert. For some time now, however, a trend toward the use of multiple experts has been emerging [1-5]. Some of the arguments raised in support of this approach include: (a) in cases where expertise is diffused and a true expert in the domain of interest cannot be identified, combining the insights of 'competent persons' could improve the application; (b) large complex domains which are generally not mastered by a single individual, require the use of multiple experts to ensure comprehensive coverage; (c) the acceptance of expert systems in the business world requires the consensus of organizational 'experts'; therefore, it is necessary to incorporate into the Expert System (ES) the contributions of several experts; and (d) larger classes of problems could be more easily solved if we move away from the notion of a single expert as the basis of an ES to the broader based 'community of experts' premise for ES applications.

It is clear that there are very strong arguments for this shift. However, it has been pointed out that we have not yet learned to deal with the problems of building ESs using a single expert, far less the increased complications of doing it with several experts $[6,7]$. One of the main problems the knowledge engineer must face is how to analyze, integrate, and verify the knowledge of multiple experts. Although several tools exist for $\mathrm{KB}$ editing and debugging, there is no methodology to support the analysis, integration and verification at the knowledge acquisition phase of ES development. Several researchers have pointed out the need for consistency analysis and validity checking in the early phases of the development process $[8,9]$. Postponing this analysis to later phases of development is costly and results in significant debugging and modification difficulties with few systems being fully verified $[10,11]$. Inasmuch as it is not possible to identify and remove all errors during the knowledge acquisition process, the knowledge engineer can benefit from knowing about potential problems. A new methodology is necessary to address effectively the issues of the multiple expert approach to ES development [12]. The focus of this paper is on providing a formal method for the analysis and integration of the rule sets of multiple experts, which could aid in identifying potential inconsistencies and redundancy problems early in the development process.

†T whom correspondence should be addressed. 


\section{PROBLEMS IN INTEGRATING THE KNOWLEDGE OF MULTIPLE EXPERTS}

Several strategies for using multiple experts in ES development have been proposed. Greenwell [13] suggests that one expert should be selected for the system design activity, and the others be involved in validating the system. Garvey et al. [2] feel that knowledge of several specialists who are more competent in specific contexts should be merged. In line with this idea, LeClair [4] developed a system which provided users with a mechanism to choose among the opinions of experts. A similar approach was also taken in the prospector system [3]. Boose [5] has proposed an approach for combining the expertise of several individuals by utilizing a common grid via the Expertise Transfer System (ETS). Others have approached the problem from the point of view of autonomous ESs co-ordinating on problem solving [1, 14-16]. The most common approaches however, attempt to obtain consensus among the experts during the knowledge acquisition phase. This is by no means a simple task, as the merging of diverse reasoning strategies becomes more error prone as the number of experts increases. Identifying potential conflicts becomes more important, because the cost of correcting errors increases exponentially as development proceeds through the life cycle.

Recently Gragun and Steudel [17] have proposed an algorithm for transforming a rule-base into a decision table and splitting the table into context-groups for analysis. Puuronen [18] has also proposed a similar approach. However, both approaches are limited with regard to rule-set integration and validation early in the life-cycle because they focus on rule-base debugging. The method we propose here is more general and flexible: (1) it targets the knowledge acquisition phase of the development life cycle; (2) it can deal with development situations where more than one domain expert is used; (3) it can be used to merge two or more rule-based ES into one comprehensive ES; and (4) it is validated with formal proofs.

\section{PRODUCTION RULES AND DECISION TABLE CONCEPTS}

Although many techniques exist for describing and representing the knowledge of experts, however, production rules are among the most popularly used because they are easier to understand and code. ESs using this technique are generally called rule-based systems [19-21]. Production rules were originally proposed by Post and were subsequently investigated and implemented in the General Problem Solver by Newell and Simon [22,23]. Every production rule consists of a condition part, which consists of one or more attributes, and action statement. There are two types of attributes; single-valued and multi-valued attributes; the former generally contain mutually exclusive values while the latter are not limited to mutually exclusive values. The second part of a production rule is the action statement, or imperative, which gets executed if the condition requirement is fulfilled. The action statement may consist of active procedures that perform operations on the knowledge base. The operations may be activating, inactivating, altering, deleting, or adding one or more rules in the knowledge base. Associated with each rule is a certainty factor, a kind of truth value which gives a numerical estimate of the experts confidence in the validity of the conclusion derived by the rule.

Decision tables (DT) have been used for decades to describe and document decision rules. Over the years a formal language has evolved for defining and analyzing DTs [24-26]. A DT is generally divided into two regions; one which specifies condition sets, and the other action sets to be executed when corresponding condition sets are satisfied. The condition sets are placed above the action sets in vertical orientation for readability. A matrix of binary entries is placed in each of the regions to indicate the condition and action specifications which define columns of rules (Fig. 1).

It should be clear that DTs can describe production rules. The condition part can be specified in the condition region of the table and the action part the action region.

\section{FORMAL DESCRIPTION OF THE METHOD}

The method is based on DT approach to describe mathematically, analyze and merge production rules via matrix methods. It focuses on four classes of problems (1) rule redundancy, (2) rule 


\begin{tabular}{|c|ccccccccc|}
\hline & \multicolumn{10}{|c|}{ DECISION RULES } \\
\cline { 2 - 9 } Conditions & $\mathbf{R} 1$ & $\mathbf{R 2}$ & $\mathbf{R 3}$ & $\mathbf{R 4}$ & $\mathbf{R 5}$ & $\mathbf{R 6}$ & $\mathbf{R 7}$ & $\mathbf{R 8}$ & R9 \\
\hline$c_{1}$ & 1 & 0 & 0 & 0 & 0 & 0 & 0 & 0 & 0 \\
$c_{1}$ & 0 & 0 & 0 & 0 & 1 & 0 & 0 & 0 & 0 \\
$c_{n}$ & 0 & 0 & 0 & 0 & 0 & 0 & 0 & 0 & 1 \\
\hline Actions & & & & & & & & & \\
\hline$a_{1}$ & 1 & 0 & 0 & 0 & 0 & 0 & 0 & 0 & 0 \\
$a_{2}$ & 0 & 0 & 0 & 0 & 1 & 0 & 0 & 0 & 0 \\
$a_{n}$ & 0 & 0 & 0 & 0 & 0 & 0 & 0 & 0 & 1 \\
\hline
\end{tabular}

Fig. 1. The structure of a DT.

inconsistency, (3) logical incompleteness of rules, and (4) merging the rules of multiple experts. Two types of redundancies can be identified: (a) logical equivalence - where the condition and action parts of two or more rules are identical; and (b) logical inclusion-where the condition of one or more rules are subjects of the condition part of one or more rules and all the rules have identical action parts. Three types of inconsistencies can be identified: (a) condition inconsistency-where two or more rules have equivalent action parts but different condition parts; (b) action inconsistency -here two or more rules have logically equivalent condition parts but different action parts; and (c) dynamic--here during processing of the rule-base, rules may develop any of the above types of inconsistencies. Although we will provide a formal description of this problem in the following, its solution is beyond the scope of this paper.

Before we enter the discussion on method, it is necessary to present some definitions, concepts and propositions.

\subsection{Definitions and notation}

$\begin{array}{cl}\text { Notation } & \text { Definition of Concept } \\ c_{k} & \text { An elementary condition is a unit condition that cannot be decomposed } \\ \text { into simpler conditions. } \\ a_{R} \quad \begin{array}{l}\text { An elementary action is a unit action that cannot be decomposed into } \\ \text { simpler actions. }\end{array} \\ C_{i} \quad \text { A complex condition consists of a conjunction involving at least one } \\ \text { elementary condition. } \\ A_{j} \quad \text { A complex action consists of a disjunction involving at least one } \\ R_{i j} \quad \begin{array}{l}\text { elementary action. } \\ A_{j} ; R_{i j}=\left(C_{i}, A_{j}\right) \text {. }\end{array}\end{array}$

$D^{E t}=\left\{d_{i j}^{E t}\right\} a 0-1$ decision table matrix for expert $E^{t}$, such that $d_{i j}^{E t}=1$ if rule $R_{i j}$ was proposed by expert $E^{t}$ and $d_{i j}^{E t}=0$ if rule $R_{i j}$ was not proposed by expert $E^{\prime}$.

\subsection{Proposition}

In the following, we formally define a set of five propositions that refer to the four classes of problems which our method is addressing.

Proposition 1. Redundancy: logical equivalence

Let $R_{i 1 j 1}=\left(C_{i 1}, A_{j 1}\right), R_{i 2 j 2}=\left(C_{i 2}, A_{j 2}\right)$ and $A_{j 1} \equiv A_{j 2}$ and $C_{i 1} \equiv C_{j 2}$. Then $\left[\left\{C_{i 1}\right.\right.$ or $\left.C_{i 2}\right) \Rightarrow\left\{A_{i 1}\right.$ or $\left.A_{i 2}\right\}$ ]. Therefore $R_{i 1 j 1} \equiv R_{i 2 i 2}$. 
Proposition 2. Redundancy: logical inclusion

Let $R_{i 1 j 1}=\left(C_{i 1}, A_{j 1}\right), R_{21 j 2}=\left(C_{21}, A_{j 2}\right)$ be valid rules, where $A_{j 1}$ is equivalent to $A_{j 2}$, and $C_{i 1}$ is a superset of $C_{i 2}$, Then $R_{i 2 j 2}$ is logically included in $R_{i 1 j 1}$.

Proof. Since $C_{i 1}$ is a superset of $C_{i 2}$ then $C_{i 1}$ is true whenever $C_{i 2}$ is true. Therefore the rule $R_{i 2 j 1}=\left(C_{i 2}, A_{j 1}\right)$ is logically determined by rule $R_{i 1 j 1} . R_{i 2 j 1}$ may therefore be said to be logically included in rule $R_{i 1 j 1}$. But since action $A_{j 1}$ is equivalent to action $A_{j 2}$, then rule $R_{i 2 j 1}$ is equivalent to rule $R_{i 2 j 2}$. Therefore rule $R_{i 2 j 2}$ is logically included in $R_{i 1 j 1}$.

Proposition 3. Action inconsistency

Given rules $R_{i 1 j 1}=\left(C_{i 1}, A_{j 1}\right), R_{21 j 2}=\left(C_{21}, A_{j 2}\right)$ where action $A_{j 1}$ conflicts with action $A_{j 2}$, and $C_{i 1}$ is a superset of $C_{i 2}$, then the pair of rules conflict.

Proof. Since $C_{i 1}$ is a superset of $C_{i 2}$ then condition $C_{i 1}$ is true whenever condition $C_{i 2}$ is true. Hence the rule $R_{i 2 j 1}=\left(C_{i 2}, A_{j i}\right)$ can be logically derived from the rule $R_{i \mid j 1}$. The result is the super rule $C_{i 2} \rightarrow\left(A_{j 1}\right.$ and $\left.A_{j 2}\right)$. But since $A_{j 1}$ and $A_{j 2}$ conflict then this rule is inconsistent.

Proposition 4. Condition inconsistencies

Let $R_{i 1 j 1}=\left(C_{i 1}, A_{j 1}\right), R_{i 2 j 2}=\left(C_{i 2}, A_{j 2}\right)$ and $C_{i 1}$ conflicts with $C_{i 2}$ and $A_{j 1} \equiv A_{j 2}$. Then $\left[C_{i 1}\right.$ or $C_{i 2} \Rightarrow\left\{A_{j 1}\right.$ and $\left.\left.A_{j 2}\right]\right\}$.

Proof. Since $A_{j 1}=A_{j 2}$ whenever $A_{j 1}$ is implied $A_{j 2}$ is also implied.

Proposition 5. Merging multiple rule sets

For the sake of clarity, we will discuss the merging of rules of different experts. However, the method is general and can be applied to rule-bases without modification.

Let $D^{E_{1}}, D^{E_{2}}$ be DT matrices of rules proposed by experts $E_{1}$ and $E_{2}$, respectively. The $D^{E_{12}}=D^{E_{1}}+D^{E_{2}}$ is a DT matrix which represents a combination of the rules proposed by experts $E_{1}$ and $E_{2}$, where $d_{i j}^{E} \geqslant 1$ indicates that rule $R_{i j}$ was proposed by either experts $E_{1}$ or $E_{2}$ and $d_{i j}^{E_{12}}=0$ indicates that $R_{i j}$ was not proposed by either of the two experts.

Proof. By definition the matrices $D^{E_{1}}, D^{E_{2}}$ and $D^{E_{12}}$ have the same dimensions and,

$$
d_{i j}^{E_{12}}=d_{i j}^{E_{1}}+d_{i j}^{E_{2}} \text {. }
$$

Thus

$$
\begin{aligned}
& d_{i j}^{E_{12}} \geqslant 1 \Rightarrow d_{i j}^{E_{1}} \geqslant 1 \quad \text { and } / \text { or } \quad d_{i j}^{E_{2}} \geqslant 1, \\
& d_{i j}^{E_{12}}=0 \Rightarrow d_{i j}^{E_{1}}=0 \quad \text { and } \quad d_{i j}^{E_{2}}=0 .
\end{aligned}
$$

Merging the rules of $T$ experts: let $T$ be the number of experts proposing rules and $D^{E^{t}}$ be the DT of rules of expert $E_{t}$. Then $\tilde{D}^{E}=\Sigma_{t=1}^{T} D^{E_{t}}$ is the DT matrix of rules proposed by all experts such that $\partial_{i j}^{E} \geqslant 1$ if and only if rule $R_{i j}$ was proposed by at least one expert and $\partial_{i j}^{E}=0$ if rule $R_{i j}$ was not proposed by any of the $T$ experts.

Now let $D^{E}=\left\{d_{i j}\right\}$ such that

$$
\begin{array}{llll}
d_{i j}=1 & \text { if } & d_{i j}^{E}>1 \\
d_{i j}=0 & \text { if } & d_{i j}^{E}>0 .
\end{array}
$$

Thus $D^{E}$ is a DT matrix such that $d_{i j}=1$ if rule $R_{i j}$ was proposed by at least one expert.

\section{PROCEDURE FOR PREPARING AND ANALYZING DECISION MATRICES}

For each expert $E_{t}$ a $0-1$ decision matrix $\left\{D^{E_{t}}\right\}$ needs to be prepared for analysis. The approach taken is to examine the rules and define from these condition sets $\left\{S_{C}\right\}$ and action sets $\left\{S_{A}\right\}$ with associated index values, then create the matrix. For the discussion, we will use two of the six rule sets taken from a real-world case on which we have successfully applied the method. To use the role sets of all six experts would lead to information overload and confuse our readers. Although the rule sets represent only a small part of the system which was developed, they are adequate for demonstrating the procedure. 
Our case example involves the rule sets proposed by two experts.

Rules proposed by expert 1.

$\begin{array}{llll}\text { IF }\left\{c_{1}, c_{2}, c_{3}, c_{4}, c_{5}\right\} & \text { THEN } & \left\{a_{1}\right\} ; \\ \text { IF }\left\{c_{1}, c_{2}, c_{3}, c_{6}, c_{7}\right\} & \text { THEN } & \left\{a_{2}, a_{5}, a_{8}, a_{9}\right\} ; \\ \text { IF }\left\{c_{1}, c_{2}, c_{8}, c_{6}, c_{7}\right\} & \text { THEN } & \left\{a_{3}, a_{5}, a_{8}, a_{9}\right\} ; \\ \text { IF }\left\{c_{1}, c_{9}, c_{3}, c_{4}, c_{7}\right\} & \text { THEN } & \left\{a_{3}, a_{6}, a_{8}, a_{9}\right\} ; \\ \text { IF }\left\{c_{1}, c_{9}, c_{8}, c_{4}, c_{7}\right\} & \text { THEN } & \left\{a_{2}, a_{6}, a_{8}, a_{9}\right\} ; \\ \text { IF }\left\{c_{1}, c_{9}, c_{3}, c_{6}, c_{7}\right\} & \text { THEN } & \left\{a_{3}, a_{6}, a_{8}, a_{9}\right\} ; \\ \text { IF }\left\{c_{1}, c_{10}, c_{11}, c_{4}\right\} & \text { THEN } & \left\{a_{3}, a_{6}, a_{8}, a_{9}\right\} ; \\ \text { IF }\left\{c_{1}, c_{10}, c_{11}, c_{6}\right\} & \text { THEN } & \left\{a_{4}, a_{7}\right\} ; \\ \text { IF }\left\{c_{1}, c_{10}, c_{8}, c_{4}\right\} & \text { THEN } & \left\{a_{3}, a_{6}, a_{8}, a_{9}\right\} ; \\ \text { IF }\left\{c_{1}, c_{10}, c_{8}, c_{6}\right\} & \text { THEN } & \left\{a_{3}, a_{7}\right\} ; \\ \text { IF }\left\{c_{1}, c_{10}, c_{3}, c_{6}\right\} & \text { THEN } & \left\{a_{3}, a_{5}, a_{8}, a_{9}\right\} ; \\ \text { IF }\left\{c_{1}, c_{10}, c_{3}, c_{4}\right\} & \text { THEN } & \left\{a_{2}, a_{5}, a_{8}, a_{9}\right\} ;\end{array}$

Rules proposed by expert 2 .

$\begin{array}{llll}\text { IF }\left\{c_{1}, c_{9}, c_{3}, c_{4}, c_{7}\right\} & \text { THEN } & \left\{a_{3}, a_{6}, a_{8}, a_{9}\right\} ; \\ \text { IF }\left\{c_{1}, c_{9}, c_{8}, c_{4}, c_{7}\right\} & \text { THEN } & \left\{a_{3}, a_{6}, a_{8}, a_{8}\right\} ; \\ \text { IF }\left\{c_{1}, c_{9}, c_{3}, c_{6}, c_{7}\right\} & \text { THEN } & \left\{a_{3}, a_{6}, a_{8}, a_{9}\right\} ; \\ \text { IF }\left\{c_{1}, c_{10}, c_{11}, c_{4}\right\} & \text { THEN } & \left\{a_{3}, a_{6}, a_{8}, a_{9}\right\} ; \\ \text { IF }\left\{c_{1}, c_{10}, c_{3}, c_{4}\right\} & \text { THEN } & \left\{a_{2}, a_{6}, a_{8}, a_{9}\right\} ; \\ \text { IF }\left\{c_{1}, c_{10}, c_{3}, c_{6}\right\} & \text { THEN } & \left\{a_{3}, a_{5}, a_{8}, a_{9}\right\} ; \\ \text { IF }\left\{c_{1}, c_{10}, c_{8}\right\} & \text { THEN } & \left\{a_{3}, a_{6}\right\} ; \\ \text { IF }\left\{c_{1}, c_{2}, c_{6}, c_{7}\right\} & \text { THEN } & \left\{a_{3}, a_{6}, a_{8}, a_{9}\right\} ;\end{array}$

Now let $S_{C}$ be the set of currently identified complex conditions and their associated index values i.e. $\left(i, C_{i}\right) \in S_{C}$ where $\left|S_{C}\right|$ equals the number of elements in $S_{C}$, and let $S_{A}$ be the set of currently identified complex actions and their associated index values i.e. $\left(j, A_{j}\right) \in S_{A}$, where $\left|S_{A}\right|$ equals the number of elements in $S_{A}$.

where

$c_{1}:$ Structural distress is present;

$c_{2}$ : Load bearing capacity of structure is low;

$c_{3}$ : Structural failure risk factor is high;

$c_{4}$ : Alternative route exists;

$c_{5}$ : Rehabilitation is not feasible;

$c_{6}$ : Alternative route does not exist;

$c_{7}$ : Rehabilitation is feasible;

$c_{8}$ : Structural risk factor is medium;

$c_{9}$ : Load bearing capacity of structure is medium;

$c_{10}$ : Load bearing capacity of structure is high;

$c_{11}$ : Structural risk factor is low;

and

$a_{1}:$ Condemn the structure;

$a_{2}$ : Completely restrict traffic in rush hours;

$a_{3}$ : Partially restrict traffic in rush hours;

$a_{4}$ : Do not restrict traffic in rush hours;

$a_{5}$ : Completely restrict traffic in nonrush hours;

$a_{6}$ : Partially restrict traffic in nonrush hours;

$a_{7}$ : Do not restrict traffic in nonrush hours;

$a_{8}$ : Rehabilitate structure in rush hours;

$a_{9}$ : Rehabilitate structure in nonrush hours. 
Now let $D^{E_{t}}=\left\{d_{i j}\right\}$ be a 0-1 decision matrix of rules for the expert $E_{t}$, where $D_{i}^{E_{t}}$ is the $i$ th row and $D_{j}^{E_{t}}$ is the $j$ th column of $D^{E_{t}}$ when $d_{i j}=0$ a value which has not been explicitly assigned by the procedure. From the above, we create a decision matrix with $\left|S_{C}\right|^{*}\left|S_{A}\right|$ dimensions where the following rules are definable:

$$
\begin{aligned}
C_{i} & \Rightarrow \bigcap_{\left[j: d_{i j}=1\right]} A_{j} \\
\bigcup_{\left[j: d_{i j}=1\right]} C_{i} & \Rightarrow A_{j} .
\end{aligned}
$$

Let $X=\left\{x_{i k}\right\}$, a $\left|S_{c}\right|^{*}\left|S_{c}\right|$ dimensioned matrix, and $Y=\left\{y_{k j}\right\}$, be a $\left|S_{A}\right|^{*}\left|S_{A}\right|$ dimensioned matrix, defined as follows:

(a) $x_{i k}=2$ if $C_{i}$ is a proper subset of $C_{k}$;

$$
=1 \text { if } i=k \text {; }
$$

0 otherwise

(b) $y_{k j}=\mathrm{I}$ if $A_{j}$ and $A_{k}$ conflict;

0 otherwise.

Also let $W=\left\{w_{i j}\right\}=X^{*} D^{E}$ such that $w_{i j}=\Sigma_{k} x_{i k} d_{k j}, V=\left\{v_{i j}\right\}$ where $v_{i j}=w_{i j}\left(\Sigma_{k} w_{i k} y_{k j}\right)$, and $U=\left\{u_{i}\right\}$ be a column vector with $\left|S_{c}\right|$ rows where $U=V^{*} E, E=\left\{e_{i}\right\}$ being a conformably dimensioned unit column vector.

\subsection{Analysis of the matrices}

We will now define the set of theorems upon which our analysis of the matrices is based.

\section{Theorem 1.}

If $D_{i_{1}}^{E_{e}}=D_{i_{2}}^{E_{e}}$, then;

Proof.

$$
\left\{C_{i 1} \cup C_{i_{2}}\right\} \Rightarrow \bigcap_{\left\{j: d_{1} j=1\right\}} A_{j}
$$

But

$$
\begin{aligned}
& C_{i_{1}} \Rightarrow \bigcap_{\left\{j: d_{i_{1}} j=1\right\}} A_{j} \\
& C_{i_{2}} \Rightarrow \bigcap_{\left\{j: d_{i_{1}} j=1\right\}} A_{j} ;
\end{aligned}
$$

because $D_{i_{1} \cdot}^{E_{1}}=D_{i_{2}}^{E_{1}}$. Therefore;

$$
\bigcap_{\left\{j: d_{i}, j=1\right\}} A_{j}=\bigcap_{\left\{j: d_{i z} j=1\right\}} A_{j},
$$

$$
\left\{C_{i_{1}} \cup C_{i_{2}}\right\} \Rightarrow \bigcap_{\left\{j: d_{i} j=1\right\}} A_{j} .
$$

Theorem 2.

If $D_{\cdot j_{1}}^{E_{l}}=D_{\cdot j_{2}}^{E_{l_{2}}}$, then;

Proof.

$$
\bigcup_{\left\{i: d_{i j_{1}}=1\right\}} C_{i} \Rightarrow\left\{A_{j_{1}} \cup A_{j_{2}}\right\}
$$

$$
\begin{gathered}
\bigcup_{\left\{i: d_{i j_{1}}=1\right\}} C_{i} \Rightarrow A j_{1} \\
\bigcup_{\left\{i: d_{i j_{1}}=1\right\}} C_{i} \Rightarrow A j_{2} .
\end{gathered}
$$


But

because $D_{. J_{1}}^{E_{l_{1}}}=D_{. j_{2}}^{E_{1}}$. Therefore;

$$
\bigcup_{\left\{i: d_{i j_{1}}=1\right\}} C_{i}=\bigcup_{\left\{i: d_{i j_{1}}=1\right\}} C_{i}
$$

$$
\bigcup_{\left\{i: d_{i j_{1}}=1\right\}} C_{i} \Rightarrow\left\{A_{j_{2}} \cap A_{j_{2}}\right\}
$$

\section{Theorem 3.}

The matrix $W$ contains only non-negative values such that $w_{i j}>0$ iff rule $R_{i j}$ was proposed, and/or there is some other rule $R_{k j}$ that was proposed and this rule is logically included in $R_{i j}$.

Proof. Based on the definition of $w_{i j}$ we have:

$$
w_{i j}=d_{i j}=\sum_{(k: k \neq i)} x_{i k} d_{k j}
$$

Thus $w_{i j}$ is always nonnegative because by definition each $d_{i j}$ and $x_{k j}$ is nonnegative.

Now if rule $R_{i j}$ was proposed then $d_{i j}=1$ and $w_{i j}>=d_{i j}=1$. If a rule $R_{k j}$ was proposed such that $R_{k j}$ logically determines $R_{i j}$ then this would imply that $C_{i}$ is a subset of $C_{k}$. Thus $x_{i k}=2, d_{k j}=1$ and $w_{i j}>=x_{i k} d_{k j}=2$. If rule $R_{i j}$ was not proposed and there is no rule $R_{k j}$ (which logically determines $R_{i j}$ ) that was proposed then $d_{i j}$ and all $d_{k j}, x_{i k}$ are zero then $w_{i j}=0$.

\section{Corollary 1}

If rule $R_{i l, j}$ is logically determined by rule $R_{i 2, j}$ when $w_{i l, j}>w_{i 2, j}$.

Proof. If rule $R_{i 1, j}$ is logically determined by rule $R_{12, j}$ then $C_{i 1}$ is a subset of $C_{i 2}$ (i.e. $x_{i 1, i 2}=2$ ), and for each $C_{r}$ such that $C_{i 2}$ is a subset of $C_{r}$ (i.e. $x_{i 2, r}=2$ ) then $C_{i 1}$ is a subset of $C_{r}\left(\right.$ i.e. $x_{i 1, r}=2$ ). Thus since;

$$
\begin{aligned}
& w_{i 1, j}=d_{i 1, j}+2^{*} d_{i 2, j}+\sum_{(k: k \neq i 1, i 2)} x_{i k} d_{k j} \\
& w_{i 2, j}=d_{i 2, j}+\sum_{(k: k \neq i 1, i 2)} x_{i k} d_{k j}
\end{aligned}
$$

then $w_{i 1, j}>w_{i 2, j}$.

\section{Corollary 2}

In matrix $W, w_{i 1, j}>1$ if there is at least one $w_{i 2, j}=1$ such that $C_{i l}$ is a proper subset of $C_{i 2}$.

Proof. By definition $w_{i 1, j}>1$ implies that there is at least one $C_{r}$ that is a superset of $C_{i 1}$ (i.e. $x_{i 1, r}=2$ ) and $d_{r j}=1$. Thus for each such $C_{i 1}$ there is a maximal set of conditions, say $S_{c i 1}$, such that $C_{i 1}$ is a proper subset of each $C_{r} \in S_{c i l}$, where each $d_{r j}=1$, and there is no condition with these properties that is not included in $S_{c i l}$. Now there has to be at least one $C_{r . .}$. Hence $w_{r \cdot . j}=d_{r . . j}=1$.

\section{Corollary 3}

In matrix $W, w_{i j}$ is positive even valued number iff $R_{i j}$ was not proposed but is logically determined by at least one other rule, and $w_{i j}$ is a positive odd valued number larger than 2 iff $R_{i j}$ was proposed and is also logically determined by at least one other rule.

Proof. This follows from the definition of $w_{i j}$.

\section{Theorem 4.}

$V$ is a matrix with nonnegative values such that $v_{i j}>0$ iff there exists at least one pair of rules, $R_{i j}$ and $R_{i k}$, which conflict with each other.

Proof. Since from Proposition 5 all $w_{i k}$ are nonnegative, and by definition all $y_{k j}$ are nonnegative then $v_{i j}$ is nonnegative. 
Now since $v_{i j}=w_{i j}\left(\Sigma_{k} w_{i k} y_{k j}\right)$ then $v_{i j}>0$ iff $w_{i j}>0$ and there is at least one $k$ such that $w_{i k}>0$ and $y_{k j}=1$. But this situation implies that rules $R_{i j}$ and $R_{i k}$ both exists, and that actions $A_{j}$ and $A_{k}$ conflict. But from Proposition 3 this means that the rules $R_{i j}$ and $R_{i k}$ conflict.

\section{Theorem 5.}

$U$ is a column vector with nonnegative values such that $u_{i}>0$ iff there exists at least one pair of rules $R_{i j}$ and $R_{i k}$ which conflict with each other.

Proof. By definition $u_{i}=\Sigma_{j} w_{i j} e_{i}=\Sigma_{j} w_{i j}$, and so $u_{i}>0$ iff at least one $w_{i j}>0$. But $w_{i j}>0$ implies that there exists at least one pair of rules $R_{i j}$ and $R_{i k}$ which conflict with each other.

\subsection{Algorithm for the procedure}

The following is the algorithm which we have implemented to provide computer support for the method. For clarity and comprehensibility, we have inserted explanatory comments for each step 1 through 5.

Step 0

$$
\text { SET } \begin{aligned}
S_{C} & =0 \\
S_{A} & =0 \\
D^{E} & =0 \\
X & =0 \\
Y & =0
\end{aligned}
$$

\section{Step 1}

For each expert $E_{r}$ :

For each rule $R_{p q}=\left(C_{p}, A_{q}\right)$ :

(a) Determine the global index $i$ for $C_{p}$ by searching $S_{C}$.

If $C_{p}$ does not currently exist in $S_{c}$,

Then

Set $i=\left|S_{C}\right|+1$,

$$
\begin{aligned}
& C_{i}=C_{p}, \\
& S_{c} \leftarrow S_{i} \cup\left(i, C_{i}\right)
\end{aligned}
$$

If $C_{p}$ did not previously exist in $S_{c}$

Then

For each $C_{k}$ that currently exists in $S_{c}$

For which $C_{p}$ is a subset of $C_{k}$

Set $x_{i k}=2$;

and, for each $C_{k}$ that currently exists in $S_{c}$

For which $C_{p}$ is a superset of $C_{k}$

Then

Set $x_{k i}=2$.

(b) Determine the global index $j$ for $A_{q}$ by searching $S_{A}$.

If $A_{q}$ does not currently exist in $S_{A}$,

Then

$$
\text { Set } \begin{aligned}
j=\left|S_{A}\right|+1 d, \\
A_{j}=A_{q}, \\
S_{A} \leftarrow S_{A} \cup\left(i, A_{j}\right) .
\end{aligned}
$$

(c) Set $d_{i j}=1$.

(d) Set $x_{i 1}=1$ for all $i$ such that $1 \leqslant i \leqslant\left|S_{c}\right|$.

(e) For all $(k, j)$ such that $1 \leqslant k \leqslant j \leqslant\left|S_{A}\right|$

If $A_{k}$ is inconsistent with $A_{j}$

Then

Set $y_{k j}=1$,

$$
i_{j k}=1 \text {. }
$$




\section{Step 2}

(a) Compute the matrix $W$.

(b) Examine $W$ in order to identify each $w_{i j} \geqslant 0$ and odd. Each such value represents a rule that was proposed and also logically derived from at least one other rule. Thus there is the possibility that at least one of these rules was specified incorrectly.

(c) If any errors were identified in 2(b), then $D^{E}$ should be modified appropriately and $W$ recomputed.

Step 3

(a) Compute the matrices $V$ and $U$.

(b) For each $u_{i}>0$. Examine row $i$ of matrix $V$ in order to identify each rule $R_{i j}$ that is inconsistent with some other rule $R_{i k}$.

(c) If there are any consistencies then these should be resolved, $D^{E}$ should be modified appropriately and we should return to Step 2 .

\section{Step 4}

For each $W_{j}^{E}$ of $W^{E}$

For each $i_{1} \neq i_{2}$ such that $w_{i_{1} j}=1, w_{i_{2} j}=1$ and $C_{i_{1}}$ is a superset of $C_{i_{2}}$.

Set $w_{i_{2} j}=1$,

\section{Step 5}

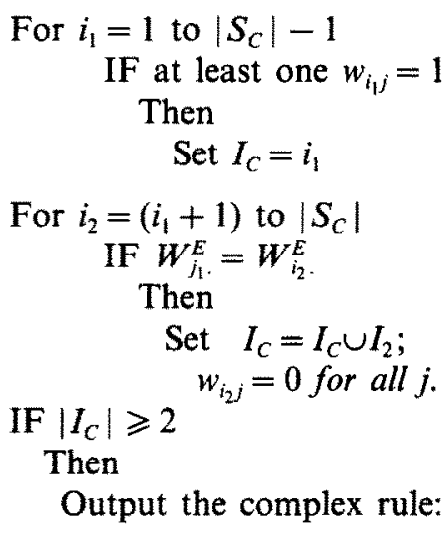

Set $w_{i_{1} j}=0$ for all $j$.

$$
\bigcup_{i I_{A}} C_{i} \rightarrow \bigcap_{\left\{j ; w_{i} j=1\right\}} A_{j}
$$

\section{Step 6}

For $j_{1}=1$ to $\left|S_{A}\right|-1$.

IF at least one $w_{i j_{1}}=1$

Then

$$
\text { Set } J_{A}=j_{1}
$$

For $j_{2}=\left(j_{1}+1\right)$ to $\left|S_{A}\right|$

IF $W_{. j_{1}}^{E}=W_{j_{2}}^{E}$

Then

$$
\text { Set } \begin{aligned}
J_{A} & =J_{A} \cup J_{2} \\
w_{i j_{2}} & =0 \text { for all } i .
\end{aligned}
$$

IF $\left|J_{A}\right|>2$

Then

Output the complex rule

Set $w_{i i_{1}}=0$ for all $i$.

$$
\bigcup_{\left\{i: w_{i j 1}\right\}} C_{i} \rightarrow \bigcap_{j K_{A}} A_{j}
$$




\subsection{The Case illustration}

In the following we walk the reader through the procedure using the rules of the case listed in Section 5 of the paper. We briefly comment on the output of each step for the sake of clarity.

\section{Output of Step 1}

\begin{tabular}{|c|c|}
\hline$s_{c}:$ The Set of Complex Conditions & $S_{A}:$ The Set of Complex Actions \\
\hline $\begin{array}{l}\left(1, c_{1}\right) \text { where } c_{1}=\left(c_{1}, c_{2}, c_{3}, c_{4}, c_{5}\right) \\
\left(2, c_{2}\right) \text { where } c_{2}=\left(c_{1}, c_{2}, c_{3}, c_{6}, c_{7}\right) \\
\left(3, c_{3}\right) \text { where } c_{3}=\left(c_{1}, c_{2}, c_{8}, c_{6}, c_{7}\right) \\
\left(4, c_{4}\right) \text { where } c_{4}=\left(c_{1}, c_{9}, c_{3}, c_{4}, c_{7}\right) \\
\left(5, c_{5}\right) \text { where } c_{5}=\left(c_{1}, c_{9}, c_{8}, c_{4}, c_{7}\right) \\
\left(6, c_{6}\right) \text { where } c_{6}=\left(c_{1}, c_{9}, c_{3}, c_{6}, c_{7}\right) \\
\left(7, c_{7}\right) \text { where } c_{7}=\left(c_{1}, c_{10}, c_{11}, c_{4}\right) \\
\left(8, c_{8}\right) \text { where } c_{8}=\left(c_{1}, c_{10}, c_{11}, c_{6}\right) \\
\left(9, c_{9}\right) \text { where } c_{9}=\left(c_{1}, c_{10}, c_{8}, c_{4}\right) \\
\left(10, c_{10}\right) \text { where } c_{10}=\left(c_{1}, c_{10}, c_{8}, c_{6}\right) \\
\left(11, c_{11}\right) \text { where } c_{11}=\left(c_{1}, c_{10}, c_{3}, c_{6}\right) \\
\left(12, c_{12}\right) \text { where } c_{12}=\left(c_{1}, c_{10}, c_{3}, c_{4}\right) \\
\left(13, c_{13}\right) \text { where } c_{13}=\left(c_{1}, c_{10}, c_{8}\right) \\
\left(14, c_{14}\right) \text { where } c_{14}=\left(c_{1}, c_{2}, c_{6}, c_{7}\right)\end{array}$ & $\begin{array}{l}\left(1, A_{1}\right) \text { where } A_{1}=\left(a_{1}\right) \\
\left(2, A_{2}\right) \text { where } A_{2}=\left(a_{2}\right) \\
\left(3, A_{3}\right) \text { where } A_{3}=\left(a_{3}\right) \\
\left(4, A_{4}\right) \text { where } A_{4}=\left(a_{5}\right) \\
\left(5, A_{5}\right) \text { where } A_{5}=\left(a_{5}\right) \\
\left(6, A_{6}\right) \text { where } A_{6}=\left(a_{6}\right) \\
\left(7, A_{7}\right) \text { where } A_{7}=\left(a_{7}\right) \\
\left(8, A_{8}\right) \text { where } A_{8}=\left(a_{8}\right) \\
\left(9, A_{9}\right) \text { where } A_{9}=\left(a_{9}\right)\end{array}$ \\
\hline
\end{tabular}

Fig. 2. Tables of condition and action sets (output of Step la and b).

\begin{tabular}{|c|c|c|c|c|c|c|c|c|c|}
\hline \multicolumn{10}{|c|}{ Matrix $D^{E}$} \\
\hline$y j$ & 1 & 2 & 3 & 4 & 5 & 6 & 7 & 8 & 9 \\
\hline 1 & 1 & 0 & 0 & 0 & 0 & 0 & 0 & 0 & 0 \\
\hline 2 & 0 & 1 & 0 & 0 & 1 & 0 & 0 & 1 & 1 \\
\hline 3 & 0 & 0 & 1 & 0 & 1 & 0 & 0 & 1 & 1 \\
\hline 4 & 0 & 0 & 1 & 0 & 0 & 1 & 0 & 1 & 1 \\
\hline 5 & 0 & 1 & 1 & 0 & 0 & 1 & 0 & 1 & 1 \\
\hline 6 & 0 & 0 & 1 & 0 & 0 & 1 & 0 & 1 & 1 \\
\hline 7 & 0 & 0 & 1 & 0 & 0 & 1 & 0 & 1 & 1 \\
\hline 8 & 0 & 0 & 0 & 1 & 0 & 0 & 1 & 0 & 0 \\
\hline 9 & 0 & 0 & 1 & 0 & 0 & 1 & 0 & 1 & 1 \\
\hline 10 & 0 & 0 & 1 & 0 & 0 & 0 & 1 & 0 & 0 \\
\hline 11 & 0 & 0 & 1 & 0 & 1 & 0 & 0 & 1 & 1 \\
\hline 12 & 0 & 1 & 0 & 0 & 1 & 1 & 0 & 1 & 1 \\
\hline 13 & 0 & 0 & 1 & 0 & 0 & 1 & 0 & 0 & 0 \\
\hline 14 & 0 & 0 & 1 & 0 & 0 & 1 & 0 & 1 & 1 \\
\hline
\end{tabular}

Fig. 3. The $0-1$ decision matrix $D^{E}$ (output of Step 1c). 


\begin{tabular}{|c|c|c|c|c|c|c|c|c|c|c|c|c|c|c|}
\hline \multicolumn{10}{|c|}{ Matrix $x$} & \multicolumn{10}{|c|}{$\mid$} \\
\hline 1 & 1 & 2 & 3 & 4 & 5 & 6 & 7 & 8 & 9 & 10 & 11 & 12 & 13 & 14 \\
\hline 2 & 1 & & & & & & & & & & & & & \\
\hline 3 & & & 1 & & & & & & & & & & & 2 \\
\hline 4 & & & & 1 & & & & & & & & & & \\
\hline 5 & & & & & 1 & & & & & & & & & \\
\hline 6 & & & & & & 1 & & & & & & & & \\
\hline 7 & & & & & & & 1 & & & & & & & \\
\hline 8 & & & & & & & & 1 & & & & & & \\
\hline 9 & & & & & & & & & 1 & & & & 2 & \\
\hline 10 & & & & & & & & & & 1 & & & 2 & \\
\hline 11 & & & & & & & & & & & 1 & & & \\
\hline 12 & & & & & & & & & & & & 1 & & \\
\hline 13 & & & & & & & & & & & & & 1 & \\
\hline 14 & & & & & & & & & & & & & & 1 \\
\hline
\end{tabular}

Fig. 4. Matrix $X$ (output of Step 1d).

We may recall that the complex condition $C_{14}=\left(c_{1}, c_{2}, c_{6}, c_{7}\right)$ is a superset of complex condition $C_{2}=\left(c_{1}, c_{2}, c_{6}, c_{7}, c_{3}\right)$ and complex condition $C_{3}=\left(c_{1}, c_{2}, c_{6}, c_{7}, c_{8}\right)$; and complex condition $C_{13}=\left(c_{1}, c_{10}, c_{8}\right)$ is a superset of complex condition $C_{9}=\left(c_{1}, c_{10}, c_{8}, c_{4}\right)$ and complex condition $C_{10}=\left(c_{1}, c_{10}, c_{8}, c_{6}\right)$.

It should be noted that action $A_{1}=$ (Condemn the structure) conflicts with the action $A_{8}=$ (Rehabilitate the structure in rush hours) and $A_{9}=$ (Rehabilitate the structure in nonrush hours). It should also be noted that each pair of actions in the set $\left\{A_{2}, A_{3}, A_{4}\right\}$ conflict where $A_{2}=$ (Completely restrict traffic in rush hours), $A_{3}=$ (Partially restrict traffic in rush hours), and $A_{4}=$ (Do not restrict traffic in rush hours). Each pair of rules in the set $\left\{A_{5}, A_{6}, A_{7}\right\}$ also conflict.

\begin{tabular}{|c|c|c|c|c|c|c|c|c|c|}
\hline \multicolumn{9}{|c|}{ Matrix $Y$} \\
\hline$y$ & 1 & 2 & 3 & 4 & 5 & 6 & 7 & 8 & 9 \\
\hline 1 & & & 1 & 1 & & 1 & 1 & 1 & 1 \\
\hline 2 & & & 1 & 1 & & & & & \\
\hline 3 & 1 & 1 & & 1 & & & & & \\
\hline 4 & 1 & 1 & 1 & & & & & 1 & \\
\hline 5 & & & & & & 1 & 1 & & \\
\hline 6 & 1 & & & & 1 & & 1 & & \\
\hline 7 & 1 & & & & 1 & 1 & & & 1 \\
\hline 8 & 1 & & & 1 & & & & & \\
\hline 9 & 1 & & & & & & 1 & & \\
\hline
\end{tabular}

Fig. 5. Matrix $Y$ (output of Step le). 
Output of Step 2 (first pass)

\begin{tabular}{|c|c|c|c|c|c|c|c|c|c|}
\hline \multicolumn{10}{|c|}{ Matrix $W$} \\
\hline$i j$ & 1 & 2 & 3 & 4 & 5 & 6 & 7 & 8 & 9 \\
\hline 1 & 1 & & & & & & & & \\
\hline 2 & & 1 & 2 & & 1 & 2 & & 3 & 3 \\
\hline 3 & & & 3 & & 1 & 2 & & 3 & 3 \\
\hline 4 & & & 1 & & & 1 & & 1 & 1 \\
\hline 5 & & 1 & 1 & & & 1 & & 1 & 1 \\
\hline 6 & & & 1 & & & 1 & & 1 & 1 \\
\hline 7 & & & 1 & & & 1 & & 1 & 1 \\
\hline 8 & & & & 1 & & & 1 & & \\
\hline 9 & & & 3 & & & 3 & & 1 & 1 \\
\hline 10 & & & 3 & & & 2 & 1 & & \\
\hline 11 & & & 1 & & 1 & & & 1 & 1 \\
\hline 12 & & 1 & & & 1 & 1 & & 1 & 1 \\
\hline 13 & & & 1 & & & 1 & & & \\
\hline 14 & & & 1 & & & 1 & & 1 & 1 \\
\hline
\end{tabular}

Fig. 6. Matrix $W$ (output of Step 2, first pass).

Output of Step 3 (first pass)

\begin{tabular}{|c|c|c|c|c|c|c|c|c|c|}
\hline \multicolumn{7}{|c|}{ Marix $V$} \\
\hline$i j$ & 1 & 2 & 3 & 4 & 5 & 6 & 7 & 8 & 9 \\
\hline 1 & & & & & & & & & \\
\hline 2 & & 2 & 2 & & 2 & 2 & & & \\
\hline 3 & & & & & 2 & 2 & & & \\
\hline 4 & & & & & & & & & \\
\hline 5 & & 1 & 1 & & & & & & \\
\hline 6 & & & & & & & & & \\
\hline 7 & & & & & & & & & \\
\hline 8 & & & & & & & & & \\
\hline 9 & & & & & & & & & \\
\hline 10 & & & & & & 2 & 2 & & \\
\hline 11 & & & & & & & & & \\
\hline 12 & & & & & 1 & 1 & & & \\
\hline 13 & & & & & & & & & \\
\hline 14 & & & & & & & & & \\
\hline
\end{tabular}

Fig. 7. Matrix $W$ (output of Step 3, first pass).

$$
U=(0,8,4,0,2,0,0,0,0,4,0,2,0,0)^{T}
$$


We observe from the entries in the vector that conflicting actions were proposed for rules in which the complex condition were $C_{2}, C_{3}, C_{10}$ and $C_{12}$.

\section{Output of Step 2 (second pass)}

\begin{tabular}{|c|c|c|c|c|c|c|c|c|c|}
\hline \multicolumn{9}{|c|}{ Marix $W$} \\
\hline $4 j$ & 1 & 2 & 3 & 4 & 5 & 6 & 7 & 8 & 9 \\
\hline 1 & 1 & & & & & & & & \\
\hline 2 & & 1 & & & 1 & & & 3 & 3 \\
\hline 3 & & & 1 & & 1 & & & 3 & 3 \\
\hline 4 & & & 1 & & & 1 & & 1 & 1 \\
\hline 5 & & 1 & $*$ & & & 1 & & 1 & 1 \\
\hline 6 & & & 1 & & & 1 & & 1 & 1 \\
\hline 7 & & & 1 & & & 1 & & 1 & 1 \\
\hline 8 & & & & 1 & & & 1 & & \\
\hline 9 & & & 1 & & & 1 & & 1 & 1 \\
\hline 10 & & & 1 & & & 0 & 1 & & \\
\hline 11 & & & 1 & & 1 & & & 1 & 1 \\
\hline 12 & & 1 & & & 1 & $*$ & & 1 & 1 \\
\hline 13 & & & $*$ & & & $*$ & & & \\
\hline 14 & & & $*$ & & & $*$ & & 1 & 1 \\
\hline
\end{tabular}

Fig. 8. Matrix $W$ (output of Step 2, second pass).

After discussions between the pair of experts, rules with the asterisk ("*") in the relevant cells of the $W$-matrix were removed.

Output of Step 3 (second pass)

$$
\begin{gathered}
U=(0,0,0,0,0,0,0,0,0,0,0,0,0,0)^{T} \\
\text { VECTOR } U
\end{gathered}
$$

The $V$-matrix was recomputed using the $W$-matrix of the second pass of Step 2, and the $U$-vector was then recomputed. We note that each entry in this vector is zero, thus indicating that there are no conflicting rules.

Output of Step 4

\begin{tabular}{|c|c|c|c|c|c|c|c|c|c|}
\hline \multicolumn{9}{|c|}{ Matrix $W$} \\
\hline$i j$ & 1 & 2 & 3 & 4 & 5 & 6 & 7 & 8 & 9 \\
\hline 1 & 1 & & & & & & & & \\
\hline 2 & & 1 & & & 1 & & & 40 & 40 \\
\hline 3 & & & 1 & & 1 & & & 40 & 40 \\
\hline 4 & & & 1 & & & 1 & & 1 & 1 \\
\hline 5 & & 1 & & & & 1 & & 1 & 1 \\
\hline 6 & & & 1 & & & 1 & & 1 & 1 \\
\hline 7 & & & 1 & & & 1 & & 1 & 1 \\
\hline 8 & & & & 1 & & & 1 & & \\
\hline 9 & & & 1 & & & 1 & & 1 & 1 \\
\hline 10 & & & 1 & & & 0 & 1 & & \\
\hline 11 & & & 1 & & 1 & & & 1 & 1 \\
\hline 12 & & 1 & & & 1 & & & 1 & 1 \\
\hline 13 & & & & & & & & & \\
\hline 14 & & & & & & & & 1 & 1 \\
\hline
\end{tabular}

Fig. 9. Matrix $W$ (output of Step 4). 
In this step we remove the rules that are logically included in another rule. The cells with the entry "No. 0" correspond to those rules which are logically included in an existing rule. Thus for example the rule $R_{2,8}=\left(C_{2}, A_{8}\right)$ is logically included in the existing rule $R_{14,8}=\left(C_{14}, A_{8}\right)$.

Output of Step 5

\begin{tabular}{|c|c|c|c|c|c|c|c|c|c|}
\hline \multicolumn{10}{|c|}{ Matrix $W$} \\
\hline ili & 1 & 2 & 3 & 4 & 5 & 6 & 7 & 8 & 9 \\
\hline 1 & 1 & & & & & & & & \\
\hline 2 & & 1 & & & 1 & & & 0 & 0 \\
\hline 3 & & & 1 & & 1 & & & 0 & 0 \\
\hline 4 & & & 10 & & & 10 & & $\oplus 0$ & $\because 0$ \\
\hline 5 & & 1 & & & & 1 & & 1 & 1 \\
\hline 6 & & & 10 & & & 10 & & $\# 0$ & $\# 0$ \\
\hline 7 & & & $\# 0$ & & & $\$ 0$ & & $\# 0$ & $\# 0$ \\
\hline 8 & & & & 1 & & & 1 & & \\
\hline 9 & & & 10 & & & mo & & $\because 0$ & $\# 0$ \\
\hline 10 & & & 1 & & & 0 & 1 & & \\
\hline 11 & & & 1 & & 1 & & & 1 & 1 \\
\hline 12 & & 1 & & & 1 & & & 1 & 1 \\
\hline 13 & & & & & & & & . & \\
\hline 14 & & & & & & & & 1 & 1 \\
\hline
\end{tabular}

Fig. 10. Matrix $W$ (output of Step 5).

In this step we locate identical rows in the $W$-matrix. The rules with the entry "No. 0 " in the corresponding cells of the $W$-matrix are removed from the matrix and combined to form the following super-rule which is output in this step:

$$
\left\{C_{4} \text { or } C_{9} \text { or } C_{6} \text { or } C_{7}\right\} \rightarrow\left\{A_{3} \text { and } A_{6} \text { and } A_{8} \text { and } A_{9}\right\}
$$

Output of Step 6

\begin{tabular}{|c|c|c|c|c|c|c|c|c|c|}
\hline \multicolumn{9}{|c|}{ Marix $W$} \\
\hline ij & 1 & 2 & 3 & 4 & 5 & 6 & 7 & 8 & 9 \\
\hline 1 & 1 & & & & & & & & \\
\hline 2 & & 1 & & & 1 & & & & \\
\hline 3 & & & 1 & & 1 & & & & \\
\hline 4 & & & & & & & & & \\
\hline 5 & & 1 & & & & 1 & & $* 0$ & 40 \\
\hline 6 & & & & & & & & & \\
\hline 7 & & & & & & & & & \\
\hline 8 & & & & 1 & & & 1 & & \\
\hline 9 & & & & & & & & & \\
\hline 10 & & & 1 & & & & 1 & & \\
\hline 11 & & & 1 & & 1 & & & 40 & 40 \\
\hline 12 & & 1 & & & 1 & & & 40 & 40 \\
\hline 13 & & & & & & & & & \\
\hline 14 & & & & & & & & 40 & 40 \\
\hline
\end{tabular}

Fig. 11. Matrix $W$ (output of Step 6). 
In this step we identify identical columns in the $W$-matrix. Rules with the entry "No. 0 " in the corresponding cells of the matrix are removed from the matrix and combined to form the following super-rule which is output in this step:

$$
\left\{C_{5} \text { or } C_{11} \text { or } C_{12} \text { or } C_{14}\right\} \rightarrow\left\{A_{8} \text { and } A_{9}\right\}
$$

Output of Step 7

\begin{tabular}{|c|c|c|c|c|c|c|c|c|c|}
\hline \multicolumn{9}{|c|}{ Matrix W } \\
\hline$i j$ & 1 & 2 & 3 & 4 & 5 & 6 & 7 & 8 & 9 \\
\hline 1 & $\# 0$ & & & & & & & & \\
\hline 2 & & $\# 0$ & & & $\# 0$ & & & & \\
\hline 3 & & & $\# 0$ & & $\# 0$ & & & & \\
\hline 4 & & & & & & & & & \\
\hline 5 & & $\# 0$ & & & & $\# 0$ & & & \\
\hline 6 & & & & & & & & & \\
\hline 7 & & & & & & & & & \\
\hline 8 & & & & $\# 0$ & & & $\# 0$ & & \\
\hline 9 & & & & & & & & & \\
\hline 10 & & & $\# 0$ & & & & $\# 0$ & & \\
\hline 11 & & & $\# 0$ & & $\# 0$ & & & & \\
\hline 12 & & $\# 0$ & & & $\# 0$ & & & & \\
\hline 13 & & & & & & & & & \\
\hline 14 & & & & & & & & & \\
\hline
\end{tabular}

Fig. 12. Matrix $W$ (output of Step 7).

In our final step the rules with the entry "No. 0 " in the corresponding cells of the $W$-matrix arc rcmoved, and are output in this step:

$$
\begin{aligned}
& C_{1} \rightarrow\left\{A_{1}\right\} \\
& C_{2} \rightarrow\left\{A_{2} \text { and } A_{5}\right\} \\
& C_{3} \rightarrow\left\{A_{3} \text { and } A_{5}\right\} \\
& C_{5} \rightarrow\left\{A_{2} \text { and } A_{6}\right\} \\
& C_{8} \rightarrow\left\{A_{4} \text { and } A_{7}\right\} \\
& C_{10} \rightarrow\left\{A_{3} \text { and } A_{7}\right\} \\
& C_{12} \rightarrow\left\{A_{2} \text { and } A_{5}\right\}
\end{aligned}
$$

\section{CONCLUDING DISCUSSION}

In this paper we have discussed a method for the analysis and integration of the rule-sets of multiple experts involved in ES development. Although our discussion has focused on production rules, the method is general and applicable to other knowledge representation techniques, which can be transformed into DTs, for example decision trees and semantic networks.

Experience in using the method has led us to adopt a two phase strategy: in Phase I a $0-1$ decision matrix is prepared and analyzed separately for each expert. The inconsistencies and redundancies discovered are resolved by the knowledge engineer and appropriate expert before the rule-sets are merged in Phase II. We have found that this approach helps to contain the analysis at later levels.

In Phase II, the rule-sets are merged and then analyzed. Problems identified at this level are discussed and resolved in a group setting. This approach has also been effective in situations where we have had more than one knowledge-engineer working on the project. In these cases, each 
knowledge-engineer takes responsibility for implementing the two phase process with the experts assigned to them before submitting to the next level analysis.

\section{REFERENCES}

[1] D. Lenat. Beings: knowledge as interacting experts. Proc. Fourth Int. Joint Conference on Artificial Intelligence 1, $126-133$.

[2] T. Garvey, J. Lowrance and M. Fischler. An inference technique for integrating knowledge from disparate sources. Proc. Seventh Int. Joint Conf. Artificial Intelligence, 1, 319-325 (1981).

[3] R. Reboh. Extracting useful advice from conflicting expertise. Proc. Eighth Int. Joint Conference on Artificial Intelligence 1, 319-325 (1983).

[4] S. LeClair. Justification of advanced manufacturing technology using expert systems. In Expert Systems (Edited by N. Botten and 1. Raz), pp. 191-199. Industrial Engineering and Management Press, Norcross, GA (1985).

[5] J. Boose. Rapid acquisition and combination of knowledge from multiple experts in the same domain. Future Computing Systems 1/2, 191-216 (1986).

6] S. Mittal and C. Dym. Knowledge acquisition from multiple experts. The AI Magazine 6(2), 3236 (1985).

[7] B. Woolf. Multiple knowledge sources in intelligent teaching systems. IEEE Expert 2(2), 41-53 (1987).

[8] J. Gashnig, P. Klahar, H. Pople, E. Shortlife and A. Terry. Evaluation of expert systems: Issues and case studies. In Building Expert Systems (Edited by F Hayes-Roth, D. A. Waterman and D. B. Lenat), pp. 241-280. AddisonWesley, Reading, MA (1983).

[9] S. M. Weiss and C. A. Kulikowski. A Practical Guide to Designing Expert Systems. Chapman and Hall London (1984).

[10] F. Brooks. No silver-bullets. IEEE Computer 10-19 April (1987).

[11] J. Geissman and R. Schultz. Verification and validation of expert systems. Al Expert 3(3), 26-33 (1988).

[12] D. Botten et al. Knowledge: integration and partitioning. Int. J. Operational Res. 23(4) (1989).

[13] M. Greenwell. Knowledge Engineering for Expert Systems. Ellis Horwood, Chichester (1988).

[14] P. Nii. The blackboard model of problem-solving. The AI Magazine 6(2), 38-53 (1986).

[15] M. Evans. Knowledge-based model of distributed problem-solving. Ph.D. Dissertation, University of Manitoba (1988).

[16] R. Kaula. An approach to open intelligent information systems. Ph.D. Dissertation, State University of New York, 19-28 (1962).

[17] B. J. Gragun and H. J. Studel. A decision-table based processor for checking completeness and consistency in rule-based expert-systems. Int. J. Man-Machine Studies 26(5), 633-648 (1987).

[18] S. Puuronen. Towards help for consistency checking during the knowledge programming of a rule-base. In The Report of the 10th IRIS Seminar, Vol. 27, pp. 641-659 (Edited by P. Jarvinen). Acta Universitas Tamperensis ser B, University of Tampere (1987)

[19] F. Hayes-Roth. Rule-based systems. Commun. ACM 28(9), 921-932 (1985).

[20] D. A. Waterman. A Guide to Expert Systems. Addison-Wesley, Reading, MA (1986).

[21] P. H. Winston. Artificial Intelligence, 2nd. edn. Addison-Wesley, Reading, MA (1984).

[22] E. Post. Formal reductions of the general combinational problem. Am. J. Math. 65, 197-268 (1943).

[23] A. Newell and H. A. Simon. Human Problem Solving. Prentice-Hall, Englewood Cliffs. NJ (1972).

[24] B. Grad. Structure and concept of decision tables. Proc. Decision Tables Symposium, New York, 19-28 (1962).

[25] P. Dixon. Decision tables and their application. Computers and Automation 13 (4), 14-19 (1964).

[26] K. Metzner and D. Barnes. Decision Table Languages. Wiley, NY (1977). 